\title{
24-hour IOP fluctuation: myth or reality?
}

Luciano Quaranta ${ }^{1}$, Ivano Riva ${ }^{1}$, Francesco Oddone ${ }^{2}$

${ }^{1}$ Department of Medical and Surgical Specialties, Radiological Sciences and Public Health, University of Brescia, Brescia, Italy; ${ }^{2} I R C C S$ Fondazione G.B. Bietti, Rome, Italy

\section{Abstract}

Existing literature is divided on the importance of short-term intraocular pressure fluctuation as an independent factor for glaucoma development and progression. In this paper we present evidences in favor of and against the value of 24-hour intraocular pressure fluctuation in the evaluation and prognosis of patients with glaucoma. Potential directions for future studies and the role of new instruments for continuous intraocular pressure monitoring will be presented.

Intraocular pressure (IOP) is an important factor in diagnosing and managing glaucoma. Studies suggest that IOP tends to fluctuate throughout the day and over longer intervals. ${ }^{1-4}$ Although mean IOP is known to correlate with glaucoma progression, ${ }^{5-8}$ actually no conclusive evidence can be drawn about IOP fluctuations.

The rationale for IOP measurements throughout the 24-hour cycle is that IOP exhibits time-dependent variation that can reach up to $6 \mathrm{mmHg}$ over a 24-hour period in healthy eyes, even more in eyes with glaucoma. ${ }^{9-12}$ Therefore, a single office-hour IOP measurement offers little information regarding the IOP profile of a patient. IOP variation could be associated with optic nerve injury because, at least in principle, the continuous and excessive fluctuation of parameters in any biological system may overwhelm the homeostatic mechanisms responsible for buffering stresses.

The traditional view is that IOP is generally higher in the morning. Konstas et al. found that although peak IOPs in up to $45 \%$ of untreated exfoliation glaucoma and $22.5 \%$ of untreated primary open-angle glaucoma (POAG) patients are outside office hours, ${ }^{13}$ mean peak IOP in 24-hour curves is generally between 6 AM and 10

Correspondence: Luciano Quaranta, USVD “Centro per lo Studio del Glaucoma”, Piazzale Spedali Civili, 1, 25123 Brescia, Italy. E-mail: luciano.quaranta@unibs.it 
AM. ${ }^{14-16}$ Quaranta et al. found similar IOP profiles (Fig. 1), ${ }^{17-19}$ but other authors did not. ${ }^{20-22}$ Discrepancies in the observed circadian IOP patterns among studies may be explained by differences in equipment (pneumotonometer vs Perkins or Goldmann tonometers) or study samples. The diagnosis of glaucoma and the use of topical IOP-lowering medication could per se influence 24-hour IOP rhythms. Moreover, age differences need to be taken into account when comparing IOP curves derived from different studies. Mansouri et al..$^{23}$ found that older healthy individuals in a sleep laboratory, irrespective of body posture, had a mean cosine-fitted peak IOP at around 10:20 AM, whereas the respective peak for younger healthy individuals was earlier, between 5:30 AM and 6:30 AM depending on body posture. Sleep lab conditions may also create an environment that affects biological rhythms. Contrary to hospital-based investigations, sleep laboratory studies may allow for some adjustment to the patient's usual routine of food intake or activities in the sitting or recumbent positions better simulating normal life.

Different levels of evidence regarding the role of IOP characteristics in glaucoma can be found in several reports. ${ }^{24-30}$ Large, well-designed, prospective studies on the importance of circadian IOP fluctuation are currently lacking, and the existing literature has not produced consistent results. Moreover, actually there is no consensus about the way to define short-term IOP fluctuation: while it's generally defined as the difference between peak and through, ${ }^{3}$ standard deviation (SD) of measurements has also been advocated. ${ }^{31}$

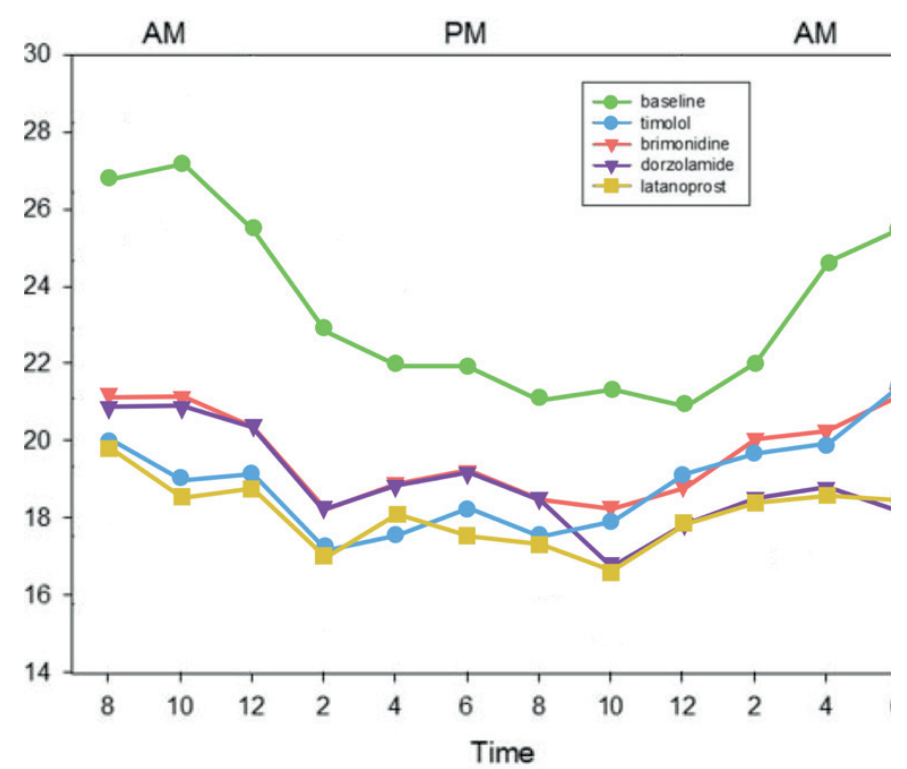

Fig. 1. 24-hour IOP profiles in treated POAG patients. (Adapted from Quaranta et al. ${ }^{17}$ ) 
Several reports found an increased short-term IOP fluctuation in patients affected by glaucoma than in controls, prevalently during office-hour. ${ }^{32-34}$ Saccà et al. found that patients affected by POAG had a greater relative daily fluctuation (between -7 and $+9.6 \%$ ) than patients with normal-tension glaucoma (NTG, between -4.7 and $+6.4 \%$ ) or healthy eyes (between $-3.4 \%$ and $+6.9 \%$ ). ${ }^{35}$ On the other hand, following a cohort of 29 patients affected by ocular hypertension (OHT) for five years, Thomas et al. found that mean daily IOP fluctuation was $8.6 \mathrm{mmHg}$ in patients progressed to POAG $(n=4)$, compared to $5.4 \mathrm{mmHg}$ in patients not progressed. ${ }^{36}$ In agreement with these results, Asrani et al. showed a strong association between diurnal fluctuation of IOP and disease progression in 105 eyes of 64 treated POAG patients using self-tonometry. ${ }^{37}$ Both the diurnal IOP and the short-term fluctuation over multiple days were significant predictors of progression. The mean office IOP had no predictive value, and the mean home IOP showed a weak association with progression. Indeed, this study was largely criticized, due to the use of self-tonometry and no stringent criteria for visual field progression evaluation. Moreover, an abnormally high rate of visual field progression was found.

In the Handan study, 47 Chinese patients affected by POAG underwent a 24-hour IOP curve, before starting any medication. ${ }^{38}$ Mean IOP fluctuation was $6.0 \pm 2.2$ $\mathrm{mmHg}$ (range, $2-11 \mathrm{mmHg}$ ), and $72 \%$ of the patients had IOP fluctuation $\geq 5 \mathrm{mmHg}$. No correlation was found between 24-hour IOP fluctuation and Humphrey mean deviation $(M D)(r=-0.166, P=0.32)$. Interestingly, in patients with unilateral POAG, Authors found no difference in mean 24-hour IOP, peak IOP, trough IOP, or IOP fluctuation when comparing the glaucomatous eye with the nonglaucomatous eye $(P>0.05)$. Results from this study should be interpreted with caution, taking into account that all patients enrolled were from oriental ethnicity and that over $90 \%$ of subjects from Handan had IOP below the cut-off of $21 \mathrm{mmHg}$.

Jonas et al..$^{39}$ performed a retrospective chart review of 855 eyes from 458 treated patients with NTG, POAG, or OHT. They investigated the potential correlation between 24-hour IOP parameters and progression of the disease, after a mean follow-up time of 55.6 months (range 5.4-124.9 months). In a multiple Cox proportional hazards model, progression of the disease was associated with age and neuroretinal RIM area. For the POAG group specifically, only age $(p<0.001)$ was a significant prognostic factor, whereas in the NTG group, higher mean IOP $(p=0.036)$ and lower fluctuation $(p=0.045)$ were identified as predictors of disease progression. Participants were receiving topical medication that is known to reduce IOP levels and its fluctuation, and the effect of 24-hour IOP variation may have been blunted.

In a recent study by Fogagnolo et al., 52 patients affected by POAG under topical therapy were followed-up for two years, after a 24-hour IOP baseline curve. ${ }^{31}$ Authors registered visual field progression endpoint and investigated baseline IOP characteristics correlated with visual field progression. Regarding 24-hour IOP characteristics, only IOP peak was correlated to visual field progression, while 24-hour IOP fluctuation was not an independent risk factor. Indeed, 24-hour mean, peak 
and fluctuation were associated with each other and a strong correlation was found between mean and peak IOP, and between fluctuation and peak IOP.

Twenty-four-hour IOP fluctuation could be a risk factor for glaucoma patients with low IOP and could influence ocular perfusion pressure. In a small cohort of 33 patients affected by NTG, Sakata et al. found that 24-hour IOP fluctuation was negatively correlated to visual field MD at baseline. ${ }^{40}$ However, Choi et al. found opposite results in a retrospective study on 113 patients affected by NTG. ${ }^{41}$ In this study, no correlation was found at baseline between 24-hour IOP fluctuation, visual field functional variables (MD and pattern standard deviation (PSD)) and anatomical variables (scanning laser polarimetry, GDX-VCC). Only fluctuation of mean ocular perfusion pressure (MOPP) was significantly correlated with decreased MD, increased PSD, and increased Advanced Glaucoma Intervention Study scores. Besides the correlation with functional outcome variables, the model identified MOPP fluctuation as an important predictor of structural damage, such as a thinner retinal nerve fiber layer. Similarly, Sung et al. published a retrospective chart review of 101 NTG patients with at least four years of follow-up and 24-hour sitting IOP and MOPP tracings. ${ }^{42}$ Multivariate regression analysis identified baseline PSD and 24-hour MOPP fluctuations as significant predictors of visual field progression, but no correlation between VF progression and either 24-hour or follow-up IOP fluctuation was found. According to the model, each $\mathrm{mmHg}$ increase in MOPP fluctuation was associated with approximately $27 \%$ greater hazard ratio of glaucoma progression during follow-up.

As a result of all these studies, no conclusive evidences about the role of short-term IOP fluctuation in glaucoma can be drawn. Moreover, other points remain to be addressed. While $24-\mathrm{h}$ IOP monitoring may provide the most accurate measurements, it is often limited by expense and doubts persist about stability of IOP patterns and IOP fluctuation from one day to the next, or between fellow eyes. Realini et al. found fair to good agreement of IOP values at each time-point in treated POAG patients who underwent two daytime IOP curves, one week apart (intraclass correlation coefficients (ICCS) ranging from 0.45 to 0.71 in right eyes and from 0.51 to 0.71 in left eyes). ${ }^{30}$ However, poor agreement was found when IOP changes over time periods were considered (e.g., the change in IOP from $8 \mathrm{AM}$ to $10 \mathrm{AM}$ on visit 1 compared with the change in IOP from $8 \mathrm{AM}$ to $10 \mathrm{AM}$ on visit 2), with ICCS coefficients ranging from -0.08 to 0.38 in right eyes and from -0.11 to 0.36 in left eyes. These results show that IOP data collected on a single day could inadequately characterize diurnal or 24-hour IOP variability over time, making IOP curve repetition a new task to explore.

Another inherent problem with circadian IOP investigations is the assumption that awakening patients at night for IOP measurements does not significantly affect their endogenous IOP rhythm. To further compound the problem, patients are often asked to walk to a nearby slit-lamp and have their IOP measured in the sitting position. A newly developed 24-hour telemetric contact lens-embedded IOP 
sensor could allow undisturbed tonometry of glaucoma patients at home ${ }^{43}$ and may corroborate some of the existing evidence regarding the circadian IOP pattern found in sleep laboratory studies. ${ }^{20-22}$ However, data provided by this instrument are not in $\mathrm{mmHg}$ and do not correlate with IOP values in $\mathrm{mmHg}$.

In conclusion, further research is needed to establish the role of 24-hour IOP fluctuation in glaucoma, and to understand if 24-hour IOP fluctuation can influence our therapeutic decisions. Since current data suggest that repeatability of IOP change over time is uniformly poor, it's important to repeat diurnal IOP recordings in case a patient continues to deteriorate, in spite of an adequate diurnal IOP control, and in all patients with advanced disease. IOP is not a static number, but tends to fluctuate throughout the 24 hours. Mean IOP is a strong predictor of glaucomatous damage. A desired therapeutic target is therefore a uniform reduction of IOP throughout the 24 hours. A reliable method of continuous IOP measurement would be desirable, making 24-hour IOP phasing easier and opening new pathways for research.

\section{References}

1. Drance SM. The significance of the diurnal phasic variation of intraocular pressure in normal and glaucomatous eyes. Trans Can Opthalmolog Soc 1960;23:131-140.

2. Kitazawa Y, Horie T. Diurnal variation of intraocular pressure in primary open-angle glaucoma. Am J Ophthalmol 1975;79(4):557-566.

3. Lee PP, Sultan MB, Grunden JW, et al. Assessing the Importance of IOP Variables in Glaucoma Using a Modified Delphi Process. J Glaucoma 2010;19(5):281-287.

4. Quaranta L, Katsanos A, Russo A, Riva I. 24-hour intraocular pressure and ocular perfusion pressure in glaucoma. Surv Ophthalmol 2013;58(1):26-41.

5. Heijl A, Leske MC, Bengtsson B, et al. Reduction of intraocular pressure and glaucoma progression: results from the Early Manifest Glaucoma Trial. Arch Ophthalmol 2002;120(10):1268-1279.

6. The Advanced Glaucoma Intervention Study (AGIS): 7. The relationship between control of intraocular pressure and visual field deterioration.The AGIS Investigators. Am J Ophthalmol 2000;130(4):429-440.

7. De Moraes CG, Juthani VJ, Liebmann JM, et al. Risk factors for visual field progression in treated glaucoma. Arch Ophthalmol 2011;129(5):562-568.

8. Garway-Heath DF, Crabb DP, Bunce C, et al. Latanoprost for open-angle glaucoma (UKGTS): a randomised, multicentre, placebo-controlled trial. Lancet 2015;385(9975):1295-1304.

9. Drance SM. Diurnal Variation of Intraocular Pressure in Treated Glaucoma. Significance in Patients with Chronic Simple Glaucoma. Arch Ophthalmol 1963;70:302-311.

10. Duke-Elder $\mathrm{S}$. The phasic variations in the ocular tension in primary glaucoma. Am J Ophthalmol 1952;35(1):1-21.

11. Langley D, Swanljung H. Ocular tension in glaucoma simplex. Br J Ophthalmol 1951;35(8):445-458.

12. Loewen NA, Liu JH, Weinreb RN. Increased 24-hour variation of human intraocular pressure with short axial length. Invest Ophthalmol Vis Sci 2010;51(2):933-937.

13. Konstas AG, Mantziris DA, Stewart WC. Diurnal intraocular pressure in untreated exfoliation and primary open-angle glaucoma. Arch Ophthalmol 1997;115(2):182-185.

14. Konstas AG, Mantziris DA, Cate EA, Stewart WC. Effect of timolol on the diurnal intraocular pressure in exfoliation and primary open-angle glaucoma. Arch Ophthalmol 1997;115(8):975-979. 
15. Konstas AG, Lake S, Economou Al, et al. 24-Hour control with a latanoprost-timolol fixed combination vs timolol alone. Arch Ophthalmol 2006;124(11):1553-1557.

16. Konstas AG, Tsironi S, Vakalis AN, et al. Intraocular pressure control over 24 hours using travoprost and timolol fixed combination administered in the morning or evening in primary open-angle and exfoliative glaucoma. Acta Ophthalmol 2009;87(1):71-76.

17. Quaranta L, Gandolfo F, Turano R, et al. Effects of topical hypotensive drugs on circadian IOP, blood pressure, and calculated diastolic ocular perfusion pressure in patients with glaucoma. Invest Ophthalmol Vis Sci 2006;47(7):2917-2923.

18. Quaranta L, Konstas AG, Rossetti L, et al. Untreated 24-h intraocular pressures measured with Goldmann applanation tonometry vs nighttime supine pressures with Perkins applanation tonometry. Eye (Lond) 2010;24(7):1252-1258.

19. Quaranta L, Miglior S, Floriani I, et al. Effects of the timolol-dorzolamide fixed combination and latanoprost on circadian diastolic ocular perfusion pressure in glaucoma. Invest Ophthalmol Vis Sci 2008;49(10):4226-4231.

20. Liu JH, Zhang X, Kripke DF, Weinreb RN. Twenty-four-hour intraocular pressure pattern associated with early glaucomatous changes. Invest Ophthalmol Vis Sci 2003;44(4):1586-1590.

21. Liu JH, Kripke DF, Hoffman RE, et al. Nocturnal elevation of intraocular pressure in young adults. Invest Ophthalmol Vis Sci 1998;39(13):2707-2712.

22. Liu JH, Bouligny RP, Kripke DF, Weinreb RN. Nocturnal elevation of intraocular pressure is detectable in the sitting position. Invest Ophthalmol Vis Sci 2003;44(10):4439-4442.

23. Mansouri K, Weinreb RN, Liu JH. Effects of aging on 24-hour intraocular pressure measurements in sitting and supine body positions. Invest Ophthalmol Vis Sci 2012;53(1):112-116.

24. Bengtsson B, Heijl A. Diurnal IOP fluctuation: not an independent risk factor for glaucomatous visual field loss in high-risk ocular hypertension. Graefes Arch Clin Exp Ophthalmol 2005;243(6):513-518.

25. Bengtsson B, Leske MC, Hyman L, et al. Fluctuation of intraocular pressure and glaucoma progression in the early manifest glaucoma trial. Ophthalmology 2007;114(2):205-209.

26. Deokule SP, Doshi A, Vizzeri G, et al. Relationship of the 24-hour pattern of intraocular pressure with optic disc appearance in primary open-angle glaucoma. Ophthalmology 2009;116(5):833-839.

27. Gumus K, Bozkurt B, Sonmez B, et al. Diurnal variation of intraocular pressure and its correlation with retinal nerve fiber analysis in Turkish patients with exfoliation syndrome. Graefes Arch Clin Exp Ophthalmol 2006;244(2):170-176.

28. Musch DC, Gillespie BW, Niziol LM, et al. Intraocular pressure control and long-term visual field loss in the Collaborative Initial Glaucoma Treatment Study. Ophthalmology 2011;118(9):1766-1773.

29. Nouri-Mahdavi K, Hoffman D, Coleman AL, et al. Predictive factors for glaucomatous visual field progression in the Advanced Glaucoma Intervention Study. Ophthalmology 2004;111(9):1627-1635.

30. Realini T, Weinreb RN, Wisniewski S. Short-term repeatability of diurnal intraocular pressure patterns in glaucomatous individuals. Ophthalmology 2011;118(1):47-51.

31. Fogagnolo P, Orzalesi N, Centofanti M, et al. Short- and long-term phasing of intraocular pressure in stable and progressive glaucoma. Ophthalmologica 2013;230(2):87-92.

32. David R, Zangwill L, Briscoe D, et al. Diurnal intraocular pressure variations: an analysis of 690 diurnal curves. Br J Ophthalmol 1992;76(5):280-283.

33. Sihota R, Saxena R, Gogoi M, et al. A comparison of the circadian rhythm of intraocular pressure in primary phronic angle closure glaucoma, primary open angle glaucoma and normal eyes. Indian J Ophthalmol 2005;53(4):243-247.

34. Tajunisah I, Reddy SC, Fathilah J. Diurnal variation of intraocular pressure in suspected glaucoma patients and their outcome. Graefes Arch Clin Exp Ophthalmol 2007;245(12):1851-1857.

35. Sacca SC, Rolando M, Marletta A, et al. Fluctuations of intraocular pressure during the day in open-angle glaucoma, normal-tension glaucoma and normal subjects. Ophthalmologica 1998;212(2):115119.

36. Thomas R, Parikh R, George R, et al. Five-year risk of progression of ocular hypertension to primary open angle glaucoma. A population-based study. Indian J Ophthalmol 2003;51(4):329-333. 
37. Asrani S, Zeimer R, Wilensky J, et al. Large diurnal fluctuations in intraocular pressure are an independent risk factor in patients with glaucoma. J Glaucoma 2000;9(2):134-142.

38. Wang NL, Friedman DS, Zhou Q, et al. A population-based assessment of 24-hour intraocular pressure among subjects with primary open-angle glaucoma: the handan eye study. Invest Ophthalmol Vis Sci 2011;52(11):7817-7821.

39. Jonas JB, Budde WM, Stroux A, et al. Diurnal intraocular pressure profiles and progression of chronic open-angle glaucoma. Eye (Lond) 2007;21(7):948-951.

40. Sakata R, Aihara M, Murata $\mathrm{H}$, et al. Intraocular pressure change over a habitual 24-hour period after changing posture or drinking water and related factors in normal tension glaucoma. Invest Ophthalmol Vis Sci 2013;54(8):5313-5320.

41. Choi J, Kim KH, Jeong J, et al. Circadian fluctuation of mean ocular perfusion pressure is a consistent risk factor for normal-tension glaucoma. Invest Ophthalmol Vis Sci 2007;48(1):104-111.

42. Sung KR, Lee S, Park SB, et al. Twenty-four hour ocular perfusion pressure fluctuation and risk of normal-tension glaucoma progression. Invest Ophthalmol Vis Sci 2009;50(11):5266-5274.

43. Mansouri K, Shaarawy T. Continuous intraocular pressure monitoring with a wireless ocular telemetry sensor: initial clinical experience in patients with open angle glaucoma. $\mathrm{Br} \mathrm{J}$ Ophthalmol 2011;95(5):627-629. 